\title{
Fast-cooling synchrotron radiation in a decaying magnetic field and $\gamma$-ray burst emission mechanism
}

\author{
Z. Lucas Uhm ${ }^{1,2}$ and Bing Zhang ${ }^{1,2,3 \star}$
}

Synchrotron radiation of relativistic electrons is an important radiation mechanism in many astrophysical sources. In the sources where the synchrotron cooling timescale is shorter than the dynamical timescale, electrons are cooled down below the minimum injection energy. It has been believed that such 'fast cooling' electrons have a power-law distribution in energy with an index -2 , and their synchrotron radiation has a photon spectral index ${ }^{1}-1.5$. On the other hand, in a transient expanding astrophysical source, such as a $\gamma$-ray burst (GRB), the magnetic field strength in the emission region continuously decreases with radius. Here we study such a system, and find that in a certain parameter regime, the fast-cooling electrons can have a harder energy spectrum. We apply this new physical regime to GRBs, and suggest that the GRB prompt emission spectra whose low-energy photon spectral index has a typical value $^{2-5}-1$ could be due to synchrotron radiation in this moderately fast-cooling regime.

The radiation mechanism of $\gamma$-ray bursts (GMBs), the most luminous explosions in the Universe, remains unidentified after 45 years since their discovery in the late 1960s. A typical GRB prompt emission spectrum is a smoothly connected broken power law called the Band function ${ }^{2}$, whose typical low- and high-energy photon spectral indices (in the convention of $\mathrm{d} N / \mathrm{d} E_{\gamma} \propto E^{\alpha}$ or $\propto$ $E^{\beta}$ ) are $\alpha \sim-1$ and $\beta \sim-2.2$. Synchrotron radiation of electrons accelerated in relativistic shocks has been suggested as the leading mechanism ${ }^{6,7}$. However, for nominal parameters, the magnetic field strength in the GRB emission region is strong enough that the electrons are in the fast-cooling regime; that is, the cooling timescale $t_{\mathrm{c}}$ is shorter than the dynamical time scale $t_{\mathrm{dyn}}$. In this regime, it has been believed that the photon index should be -1.5 (corresponding to a spectral density distribution $F_{v} \propto v^{-1 / 2}$; ref. 1). As a result, a fast-cooling synchrotron mechanism has been disfavoured $^{8}$. Proposed solutions include introducing a spatially decaying magnetic field behind the internal shock front ${ }^{9-11}$, or slow heating from a turbulent downstream region of the shock ${ }^{12}$. Applying a synchrotron model to directly fit the GRB data has recently been carried out ${ }^{13,14}$. However, the electron cooling is not tracked to calculate a time-dependent photon spectrum in their modelling.

This well-known index $\alpha=-1.5$ can be derived from a simple argument. Let us consider a continuity equation of electrons in energy space $(\partial / \partial t)\left(\mathrm{d} N_{\mathrm{e}} / \mathrm{d} \gamma_{\mathrm{e}}\right)+\left(\partial / \partial \gamma_{\mathrm{e}}\right)\left[\dot{\gamma}_{\mathrm{e}}\left(\mathrm{d} N_{\mathrm{e}} / \mathrm{d} \gamma_{\mathrm{e}}\right)\right]=Q\left(\gamma_{\mathrm{e}}, t\right)$, where $\mathrm{d} N_{\mathrm{e}} / \mathrm{d} \gamma_{\mathrm{e}}$ is the instantaneous electron spectrum of the system at the epoch $t$, and $Q\left(\gamma_{\mathrm{e}}, t\right)$ is the source function above a minimum injection Lorentz factor $\gamma_{\mathrm{m}}$ of the electrons. For synchrotron radiation, the electron energy loss rate is

$$
\dot{\gamma}_{\mathrm{e}}=-\frac{\sigma_{\mathrm{T}} B^{2} \gamma_{\mathrm{e}}^{2}}{6 \pi m_{\mathrm{e}} c} \propto-\gamma_{\mathrm{e}}^{2} B^{2}
$$

where $\sigma_{\mathrm{T}}, m_{\mathrm{e}}$ and $c$ are Thomson cross-section, electron mass and speed of light, respectively, and $B$ is the strength of magnetic fields in the emission region. For fast cooling, electrons are cooled rapidly to an energy $\gamma_{\mathrm{c}}(t)$ (cooling energy) below the injection energy $\gamma_{\mathrm{m}}$ at time $t$. In the regime $\gamma_{\mathrm{c}}<\gamma_{\mathrm{e}}<\gamma_{\mathrm{m}}$, one has $Q\left(\gamma_{\mathrm{e}}, t\right)=0$. Also consider a steady-state system $(\partial / \partial t=0)$, then one immediately gets $\mathrm{d} N_{\mathrm{e}} / \mathrm{d} \gamma_{\mathrm{e}} \propto \gamma_{\mathrm{e}}^{-2}$; that is, the electron spectral index is $\tilde{p}=2$. The specific intensity of the synchrotron spectrum would have a spectral index ${ }^{15} s=(\tilde{p}-1) / 2=1 / 2$ (with the convention $\left.F_{v} \propto v^{-s}\right)$. The photon spectral index (defined as $\mathrm{d} N_{\gamma} / \mathrm{d} E_{\gamma} \propto E_{\gamma}^{\alpha}$, where $E_{\gamma}$ is the photon energy, and $N_{\gamma}$ is the photon number flux) would then be $\alpha=-(1+s)=-1.5$.

The above argument relies on a crucial assumption of a steady state, which is achieved when a constant $B$ is invoked. However, in a rapidly expanding source such as a GRB, the magnetic field strength in the emission region cannot be preserved as a constant. In the rest frame of a conical jet, flux conservation indicates that ${ }^{16}$ the radial (poloidal) magnetic field component decreases as $B_{r}^{\prime} \propto r^{-2}$, whereas the transverse (toroidal) magnetic field component decreases as $B_{t}^{\prime} \propto r^{-1}$. As a result, at a large radius from the central engine where $\gamma$-rays are radiated, one has a toroidal-dominated magnetic field with $B^{\prime} \propto r^{-1}$. Here, $r$ is the distance from the central engine. Considering other effects (for example, magnetic dissipation, nonconical geometry), the decay law may be described by a more general form

$$
B^{\prime}(r)=B_{0}^{\prime}\left(\frac{r}{r_{0}}\right)^{-b}
$$

We investigate a generic problem of electron fast cooling in a decreasing magnetic field delineated by equation (1), and study the synchrotron emission spectrum. To interpret the GRB prompt emission spectra, we adopt some parameters that are relevant for GRBs. To be more generic, our calculation does not specify a particular energy dissipation mechanism or particle acceleration mechanism, and hence, can apply to a variety of GRB prompt emission models such as internal shocks ${ }^{17,18}$ and internal collisioninduced magnetic reconnection and turbulence ${ }^{19}$. We consider a toy box that contains electrons and a co-moving magnetic

\footnotetext{
${ }^{1}$ Kavli Institute for Astronomy and Astrophysics, Peking University, Beijing 100871, China, ${ }^{2}$ Department of Physics and Astronomy, University of Nevada
} Las Vegas, Nevada 89154, USA, ${ }^{3}$ Department of Astronomy, School of Physics, Peking University, Beijing 100871, China. *e-mail: zhang@physics.unlv.edu 

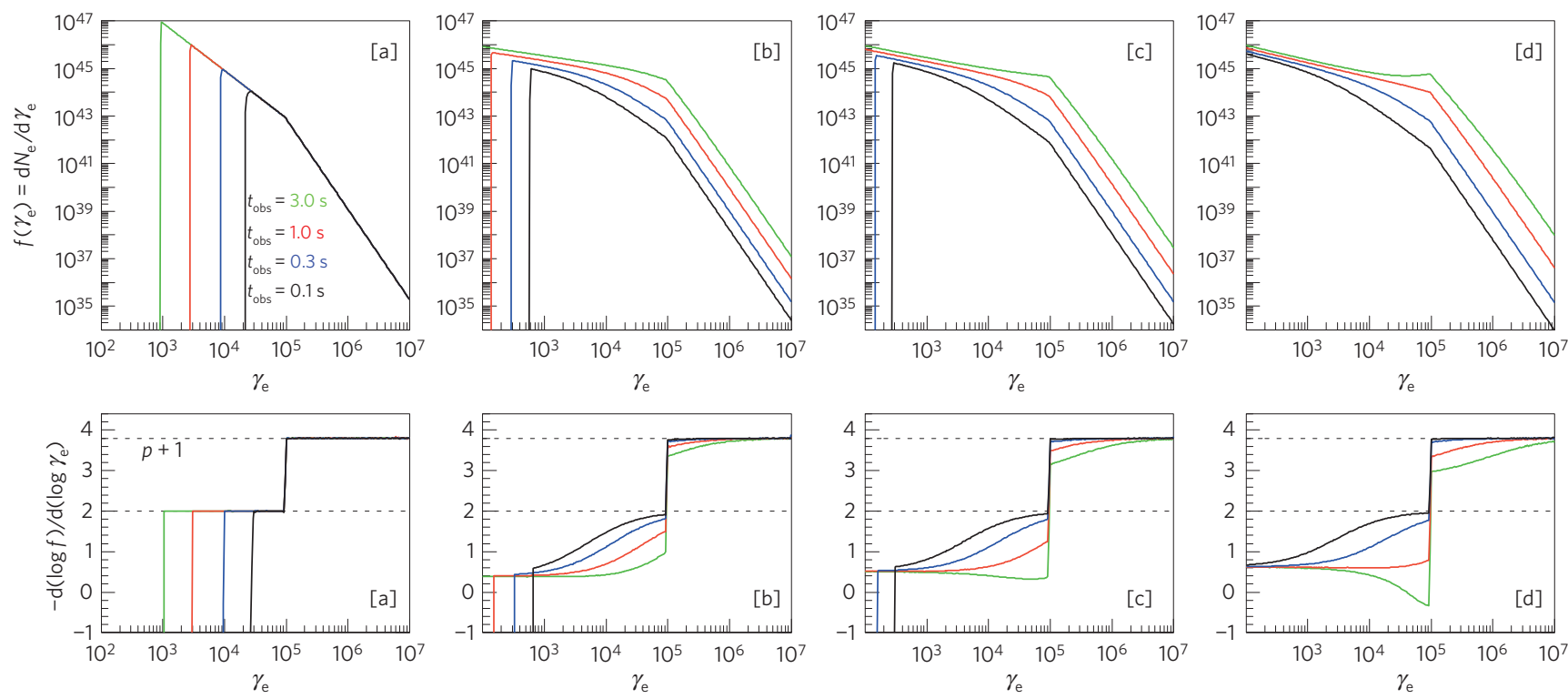

Figure 1 | The co-moving-frame fast-cooling electron energy spectrum evolution as a function of time. The injected electrons have a power-law distribution $Q\left(\gamma_{\mathrm{e}}, t^{\prime}\right)=Q_{0}\left(t^{\prime}\right)\left(\gamma_{\mathrm{e}} / \gamma_{\mathrm{m}}\right)^{-p}$ above a minimum injection Lorentz factor $\gamma_{\mathrm{m}}=10^{5}$, with a power-law index $p=2.8$ for $\gamma_{\mathrm{e}}>\gamma_{\mathrm{m}}$. We take a co-moving magnetic field $B^{\prime}=B_{0}^{\prime}\left(r / r_{0}\right)^{-b}$ with $B_{0}^{\prime}=30 \mathrm{G}$ and $r_{0}=10^{15} \mathrm{~cm}$. Four models are investigated: $b=0([a]) ; b=1([b]) ; b=1.2([\mathrm{c}]) ;$ and $b=1.5$ ([d]). For all of the models, a constant injection rate $R_{\mathrm{inj}}=\int_{\gamma_{\mathrm{m}}}^{\infty} \mathrm{Q}\left(\gamma_{\mathrm{e}}, t^{\prime}\right) d \gamma_{\mathrm{e}}=10^{47} \mathrm{~s}^{-1}$ is used, with both $Q_{0}$ and $\gamma_{\mathrm{m}}$ as constants. The electron injection into the box begins at $r=10^{14} \mathrm{~cm}$. The ejecta is assumed to be moving towards the observer with a Lorentz factor $\Gamma=300$, and the burst is assumed at a cosmological redshift $z=1$. For each model, the instantaneous electron spectra at four different epochs since the beginning of electron acceleration are calculated. The four epochs in the observer's frame are: $0.1 \mathrm{~s}$ (black), $0.3 \mathrm{~s}$ (blue), $1.0 \mathrm{~s}$ (red) and $3.0 \mathrm{~s}$ (green). For each epoch, the sharp cutoff at low energies corresponds to the 'cooling energy' of the system, which is defined by the strength of the magnetic fields and the age of the electrons. Given the same $B_{0}^{\prime}$ field at $r=10^{15} \mathrm{~cm}$, the $B^{\prime}$ field is stronger at earlier epochs, so that electrons undergo more significant cooling initially. One can see that the cooling energy is systematically lower than that of the constant $B^{\prime}$ case (model [a]) as b steepens (models [b], [c] and [d]). The lower panel of each model shows the local electron spectral index as a function of electron energy $\gamma_{\mathrm{e}}$. For model [a] (constant magnetic field), the electron spectrum shows the well-known broken power law, with the spectral indices $p+1$ and 2 above and below the injection energy, respectively. For other models, even though the index above $\gamma_{m}$ remains the same, the index below $\gamma_{m}$ is much harder. At later epochs (for example, $3 \mathrm{~s}$ spectra), the index approaches an asymptotic value $\tilde{p}_{a}=(6 b-4) /(6 b-1)$.

field $B^{\prime}$, which moves relativistically towards the observer with a bulk Lorentz factor $\Gamma$. The relativistic electrons are accelerated into a power-law distribution $Q\left(\gamma_{\mathrm{e}}, t^{\prime}\right)=Q_{0}\left(t^{\prime}\right)\left(\gamma_{\mathrm{e}} / \gamma_{\mathrm{m}}\left(t^{\prime}\right)\right)^{-p}$ (for $\gamma_{\mathrm{e}}>\gamma_{\mathrm{m}}\left(t^{\prime}\right)$ ) of a slope $p$ and continuously injected into the box at an injection rate $R_{\text {inj }}\left(t^{\prime}\right)=\int_{\gamma_{\mathrm{m}}}^{\infty} Q\left(\gamma_{\mathrm{e}}, t^{\prime}\right) \mathrm{d} \gamma_{\mathrm{e}}$, where $t^{\prime}$ is the time measured in the co-moving fluid frame. Here $R_{\text {inj }}\left(t^{\prime}\right) \delta t^{\prime}$ gives the number of electrons injected into the box during the time interval $t^{\prime}$ and $t^{\prime}+\delta t^{\prime}$.

Electrons undergo both radiative and adiabatic cooling. In the rest frame that is co-moving with the relativistic ejecta, the evolution of the Lorentz factor $\gamma_{\mathrm{e}}$ of an electron can be described by ${ }^{20}$ (noting pressure $p$ is $\propto n_{\mathrm{e}}^{4 / 3}$ in an adiabatically expanding relativistic fluid)

$$
\frac{\mathrm{d}}{\mathrm{d} t^{\prime}}\left(\frac{1}{\gamma_{\mathrm{e}}}\right)=\frac{\sigma_{T}}{6 \pi m_{\mathrm{e}} c} B^{\prime 2}-\frac{1}{3}\left(\frac{1}{\gamma_{\mathrm{e}}}\right) \frac{\mathrm{d} \ln n_{\mathrm{e}}}{\mathrm{d} t^{\prime}}
$$

For a conically expanding toy box, we take the co-moving electron number density $n_{\mathrm{e}} \propto r^{-2}$, which gives $d \ln n_{\mathrm{e}}=-2 d \ln r$. We divide the injection function $Q\left(\gamma_{\mathrm{e}}, t^{\prime}\right)$ into small divisions in time space $t^{\prime}$ and also in energy space $\gamma_{\mathrm{e}}$, and numerically follow cooling of each group of electrons (between $\left[t^{\prime}, t^{\prime}+\delta t^{\prime}\right]$ and $\left[\gamma_{\mathrm{e}}, \gamma_{\mathrm{e}}+\delta \gamma_{\mathrm{e}}\right]$ ) individually using equation (2). We then find the instantaneous global electron spectrum $d N_{\mathrm{e}} / d \gamma_{\mathrm{e}}$ of the system at any epoch.

We first consider four models with different decay indices $b$ in equation (1). The 'normalization' parameter of magnetic field decay law is taken as $B_{0}^{\prime}=30 \mathrm{G}$ at $r_{0}=10^{15} \mathrm{~cm}$, and a constant injection rate $R_{\text {inj }}=10^{47} \mathrm{~s}^{-1}$ is adopted. Model [a] takes the unphysical parameter $b=0$, that is, a constant co-moving magnetic field $B^{\prime}=B_{0}^{\prime}=30 \mathrm{G}$, to be compared with other models. It implies that there should be no change in the volume of the box. Thus, for this model we drop the adiabatic cooling term from equation (2). As shown in column 1 of Figs 1 and 2, this model gives the familiar electron spectrum $\mathrm{d} N_{\mathrm{e}} / \mathrm{d} \gamma_{\mathrm{e}} \propto \gamma_{\mathrm{e}}^{-2}$ below $\gamma_{\mathrm{m}}$, and the well-known photon spectrum $F_{v} \propto v^{-1 / 2}$ in the fast-cooling regime. One can see that the standard fast-cooling spectrum is reproduced for a steady-state system with a constant $B^{\prime}$ and $R_{\text {inj. }}$. Model [b] takes $b=1.0$ in equation (1). This is the case of free conical expansion with flux conservation (no significant magnetic dissipation). As shown in column 2 of Figs 1 and 2, the electron spectrum and the photon spectrum both harden with time. At $1.0 \mathrm{~s}$ after injecting the first group of electrons, the global electron energy spectral index deviates significantly from the $\tilde{p}=2$ nominal value, and hardens to around $\tilde{p} \sim 1$. The corresponding photon spectrum is nearly flat $\left(F_{v} \propto \nu^{0}\right)$, which corresponds to a photon index $\alpha \sim-1$, the typical low-energy photon index observed in most GRBs (refs 3,4). In columns 3 and 4 of Figs 1 and 2, we present models [c] and [d], for which steeper decay indices $b=1.2$ and $b=1.5$ are adopted, respectively. They may correspond to the cases when significant magnetic dissipation occurs during the course of synchrotron radiation. As shown in Fig. 2, both models also give spectra that are consistent with the observations.

To understand the physical origin of such an effect, in Fig. 3 we decompose the $t_{\mathrm{obs}}=1.0 \mathrm{~s}$ instantaneous electron spectrum into the contributions of 10 injection time slices, each lasting for $0.1 \mathrm{~s}$. For the constant $B^{\prime}$ case (Fig. 3a), one can see that as the electrons age, they tend to distribute more narrowly in logarithmic energy space, so that the electron number per energy bin increases. This is because in the fast-cooling regime, as time elapses, the original electrons with 

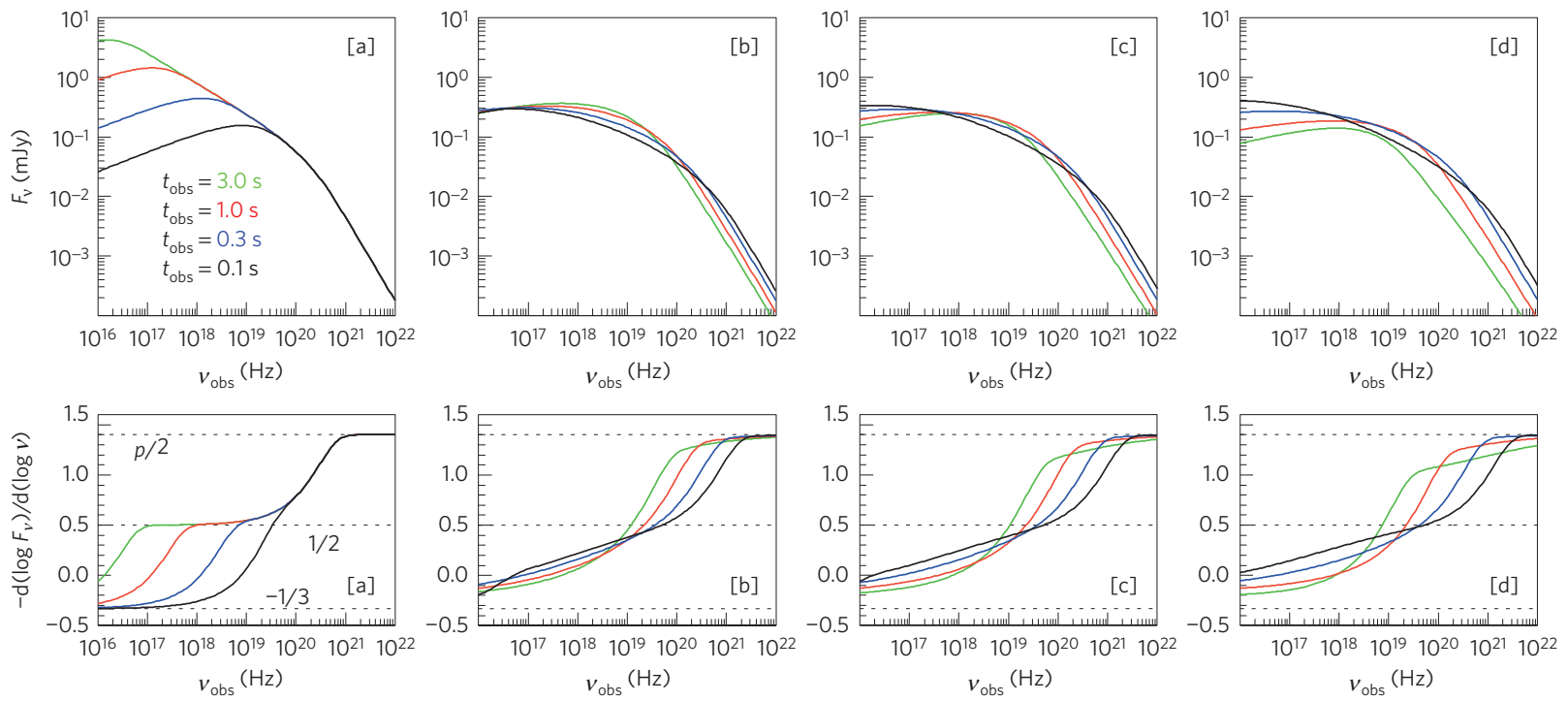

Figure 2 | The synchrotron emission flux-density $\left(F_{v}\right)$ spectra of electrons with energy distribution presented in Fig. 1. The full synchrotron spectrum of each electron ${ }^{15}$ is taken into account. The observed spectra are calculated by considering the Lorentz blueshift and cosmological redshift. Whereas the constant $B^{\prime}$ case (model [a]) gives rise to the familiar $F_{v} \propto v^{-1 / 2}$ spectrum, the decaying $B^{\prime}$ cases (models [b], [c] and [d]) all give rise to a much harder spectrum below the injection break $v_{\mathrm{m}}$. For the spectra in the seconds timescale $(1 \mathrm{~s}$, red; $3 \mathrm{~s}$, green), the low-energy spectral index is nearly flat, consistent with the typical observed photon index -1 . Lower panels show local spectral slopes as a function of observed frequency. The energy peak $E_{p}$ corresponds to the transition break towards the $p / 2$ index. Thus, a clear hard-to-soft evolution of $E_{\mathrm{p}}$ is predicted, which is consistent with the data of most broad pulses observed in $\gamma$-ray bursts (ref. 22).

a wide range of energy distribution tend to cool down to a narrower range of energy distribution defined by the ages of the electrons in the group, which are very close to each other at late epochs. Above $\gamma_{\mathrm{m}}$, the electron energy density distribution remains unchanged with time, because it is always determined by the same injection rate and cooling rate.

The cases of $B^{\prime}$ decay show a more complicated behaviour. The distribution of each group of electrons still shrinks as the group ages. However, because at early epochs the magnetic field was stronger, it had a stronger cooling effect so that for the same injection time duration $(0.1 \mathrm{~s})$, initially it had a wider spread in energy at a given age (which can be seen by comparing the $0.1 \mathrm{~s}$ electron spectrum for models [a] and [b] in Fig. 1). The later injected electrons are cooled in a weaker $B^{\prime}$ field, so that their initial spread is narrower. After the same shrinking effect due to cooling pile-up, the groups injected in earlier time slices have a wider electron distribution than the constant $B^{\prime}$ case. Also the electron spectrum above the injection energy, although possessing the same spectral index, has a normalization increasing with time owing to progressively less cooling in a progressively weaker magnetic field. These complicated effects all work in the direction to harden the spectral index, as seen in Fig. 3b,c,d. For a steeper $B^{\prime}$-decay index (for example, $b=1.2$ and $b=1.5$ ), the late-time injection occurs in an even weaker magnetic field, so that slow cooling is possible. This results in the accumulation of electrons around the minimum injection energy $\gamma_{\mathrm{m}}$, so that a sharper break in the electron energy distribution is achieved.

The model predicts that the low-energy spectrum below the injection frequency $v_{\mathrm{m}}$ is curved, owing to the complicated cooling effect as delineated in Fig. 3. Most GRB detectors have a narrow band pass so that below the peak energy (typically a few hundred kiloelectronvolts), there are at most 2 decades in energy. Nonetheless, in the detector band pass, the observed spectra are usually fitted by a Band function, with the low-energy spectral index $\alpha \sim-1$. In most situations, time-resolved spectral analyses are carried out with a time bin in seconds ${ }^{4}$. This is the typical timescale of the slow variability component in most GRB light curves ${ }^{21}$. We therefore focus on the $1 \mathrm{~s}$ and $3 \mathrm{~s}$ model spectra. We truncate these spectra in a narrow band $(5 \mathrm{keV}-5 \mathrm{MeV})$ and compare them to the empirical Band function fits (Fig. 4). One can observe that most of our model spectra are consistent with the Band function with the correct low-energy spectral indices.

Outside the band pass, our model predicts an asymptotic value of the low-energy electron energy spectral index of $\tilde{p}_{a}=(6 b-4) /(6 b-1)$, which is $2 / 5$ for $b=1$. This is seen in the numerical results of the models (lower panels in Fig. 1), and can be derived analytically (Methods). According to the simple relationship $s=(\tilde{p}-1) / 2$, one gets $s_{a}=-3 /(12 b-2)$, which is -0.3 for $b=1$ (or $F_{v} \propto v^{0.3}$ ). In reality, owing to the contribution of the $1 / 3$ segment of the individual electron spectrum, which becomes significant when $\tilde{p}$ approaches $1 / 3$ from above, the asymptotic photon spectrum limit is softened. In this case, $s$ is about -0.2 . This corresponds to a photon index of -0.8 , which is much harder than the nominal value -1.5 .

Besides the decay index $b$ as discussed above, the value of lowenergy photon index $\alpha$ also depends on several other factors: the 'normalization' parameter $B_{0}^{\prime}$ at $r_{0}=10^{15} \mathrm{~cm}$, the time history of electron injection, and the bulk Lorentz factor $\Gamma$. To see how different parameters affect the predicted $\alpha$ values, we have carried out more calculations by varying these parameters (Methods).

This model predicts a hard-to-soft evolution of the peak energy $E_{\mathrm{p}}$ during a broad pulse. This is consistent with the observational trends of a large fraction of GRBs (ref. 22). According to Figs 1 and 2, the electron spectrum also tends to harden with time, as does the $\alpha$ value. This model therefore predicts that for a broad pulse in a GRB, during the very early epochs, the $\alpha$ value would harden with time. If the $\alpha$ value of a GRB is already very hard from the very beginning, then the above-mentioned $\alpha$ evolution is no longer significant, even though the electron spectrum continues to harden with time. This is because the contributions from the $1 / 3$ spectral segment for individual electrons become more important.

Two caveats to apply this model to interpret GRB prompt emission should be noted. First, observations showed that a growing sample of GRBs have a quasi-thermal component superposed on the 

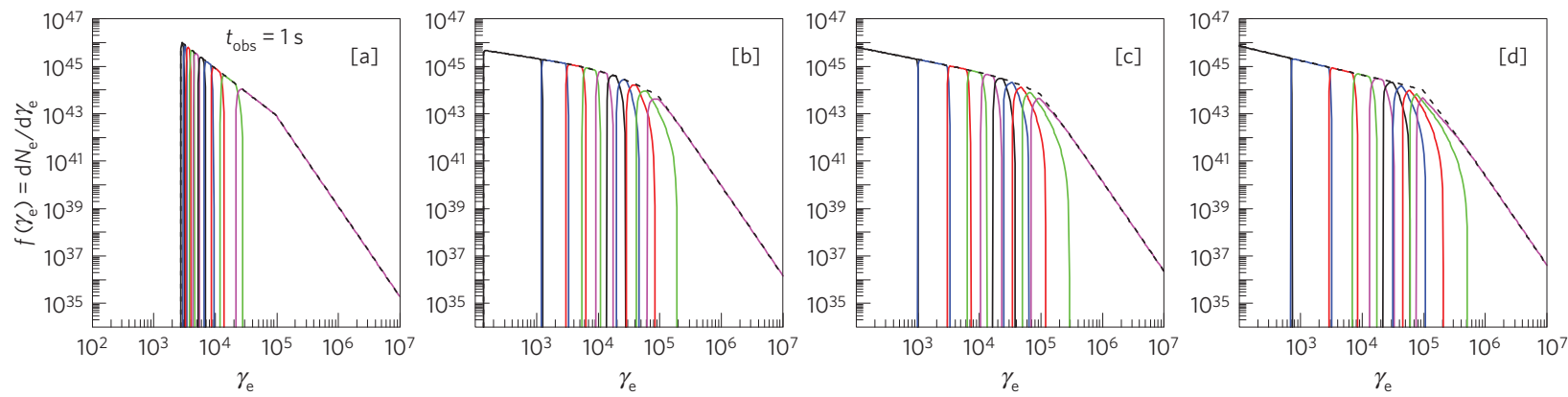

Figure 3 | Decomposition of electron spectrum at $1 \mathrm{~s}$ in the observer's frame. To see the contributions of electrons injected at different epochs, the electrons are grouped into 10 slices in injection time, each with a duration of $0.1 \mathrm{~s}$. The contributions of each group to the instantaneous electron spectrum at $1 \mathrm{~s}$ are marked in different colours. Older groups are cooled down further towards lower energies, so from left to right, curves with different colours denote the electron energy distribution of the electron groups injected from progressively later epochs, with a $0.1 \mathrm{~s}$ time step. Dashed curves are the summed total of all electrons.
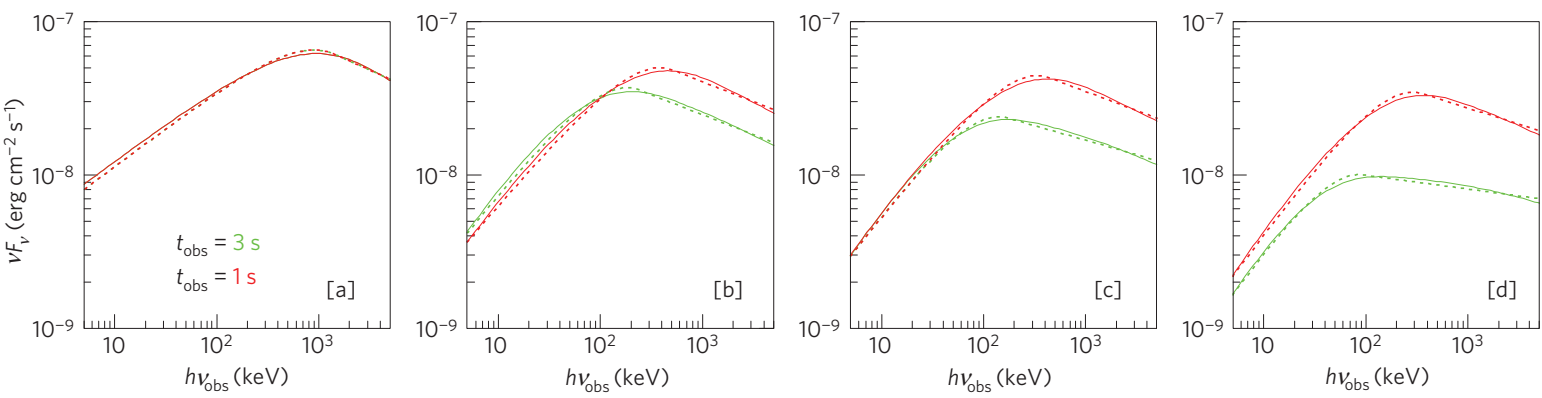

Figure 4 | A comparison of our $1 \mathrm{~s}$ and $3 \mathrm{~s}$ model spectra (solid) with the empirical Band function fits (dashed) for all four models in a narrower band pass from $5 \mathrm{keV}$ to $5 \mathrm{MeV}$. The energy spectra $\left(\nu F_{v}\right)$ are presented to show clear peak energy $\left(E_{\mathrm{p}}\right)$ in the spectra. It is seen that the model spectra can mimic the Band function spectra well. The plotted Band function parameters are the following: model [a]: $\alpha=-1.5, \beta=-2.3, E_{0}=1,800 \mathrm{keV}$ for both $1 \mathrm{~s}$ and $3 \mathrm{~s}$; model [b]: $\alpha=-1.22, \beta=-2.26, E_{0}=490 \mathrm{keV}$ for $1 \mathrm{~s}$, and $\alpha=-1.17, \beta=-2.26, E_{0}=220 \mathrm{keV}$ for $3 \mathrm{~s}$; model $[\mathrm{c}]: \alpha=-1.16, \beta=-2.25, E_{0}=400 \mathrm{keV}$ for $1 \mathrm{~s}$, and $\alpha=-1.12, \beta=-2.19, E_{0}=160 \mathrm{keV}$ for $3 \mathrm{~s}$; model $[\mathrm{d}]: \alpha=-1.1, \beta=-2.21, E_{0}=320 \mathrm{keV}$ for $1 \mathrm{~s}$, and $\alpha=-1.05, \beta=-2.09, E_{0}=90 \mathrm{keV}$ for $3 \mathrm{~s}$.

Band component ${ }^{23-25}$. Whereas the Band component is probably of a synchrotron origin ${ }^{13,26,27}$, the quasi-thermal component is widely interpreted as emission from the GRB photosphere ${ }^{28-31}$, the relative strength of which with respect to the synchrotron component depends on the composition of the GRB ejecta, and could be dominant if the ejecta is a matter-dominated fireball. As the hardest $\alpha$ value we get is about -0.8 , an observed $\alpha$ harder than this value would be evidence of a dominant photosphere component ${ }^{28}$. Second, some requirements on the parameters are needed to account for the GRB data. The observed high-energy spectral index $\beta$ requires a relatively large, yet reasonable, value of the electron injection index $p$ (for example, >2.5). More importantly, to interpret the observed $\alpha$ distribution peaking at $\alpha \sim-1$ in our model, one demands a relatively high $\gamma_{\mathrm{m}} \sim 10^{5}$ and low $B_{0}^{\prime} \sim(10-100) \mathrm{G}$. A plausible scenario to satisfy these parameter constraints may be magnetic dissipation models that invoke a large dissipation radius, such as the internal collision-induced magnetic reconnection and turbulence (ICMART) model ${ }^{19}$. Owing to the large emission radius $R \gtrsim 10^{15} \mathrm{~cm}$, this model allows secondsduration broad pulses as fundamental radiation units, during which particles are continuously accelerated. Owing to a moderately high magnetization parameter $\sigma$ in the emission region, the minimum injected electron Lorentz factor $\gamma_{\mathrm{m}} \sim 10^{5}$ can be achieved, because a small amount of electrons share a similar amount of dissipated energy. One potential difficulty is that there is a preferred range of $B_{0}^{\prime}(10-100 \mathrm{G})$ for $\alpha$ to fall into the observed distribution. The magnetization parameter

$$
\sigma=2.4 \times 10^{-4}\left(\frac{\Gamma}{300}\right)^{2}\left(\frac{B^{\prime}}{30 \mathrm{G}}\right)^{2}\left(\frac{R}{10^{15} \mathrm{~cm}}\right)^{2} L_{52}^{-1}
$$

is required to be in the range of $2.7 \times 10^{-5}-2.7 \times 10^{-3}$ for $\Gamma=300$, $R=10^{15} \mathrm{~cm}$ and $L=10^{52} \mathrm{erg} \mathrm{s}^{-1}$, which is relatively low. Within the ICMART scenario, the electrons probably radiate in the outflow region of a reconnection layer, in which magnetic fields are largely dissipated. One therefore expects a relatively low $B_{0}^{\prime}$ (and hence, low $\sigma$ ) as compared with the undissipated regions in the outflow. Nonetheless, detailed studies of magnetic reconnection and particle acceleration processes are needed to address whether the $B_{0}^{\prime}$ range demanded by the model could be achieved.

The new physics in the moderately fast-cooling regime discussed in this paper would find applications in many other astrophysical systems invoking jets and explosions, such as active galactic nuclei, galactic 'micro-quasars' in X-ray binaries and jets from tidal disruption of stars by supermassive black holes. Within the GRB context, it also finds application in the afterglow phase where electrons never enter a deep fast-cooling regime. Further investigations of this physical process in other astrophysical environment are called for.

\section{Methods}

Asymptotic value of $\alpha$. The asymptotic low-energy spectral index can be derived analytically from equation (2). Assuming a constant Lorentz factor $\Gamma$ (which is relevant for GRB prompt emission), one has $r=c t^{\prime} \Gamma$. We first solve a simpler equation by dropping the adiabatic term, that is

$$
\frac{\mathrm{d}}{\mathrm{d} t^{\prime}}\left(\frac{1}{\gamma_{\mathrm{e}}}\right)=a t^{\prime-2 b}
$$

where

$$
a \equiv \frac{\sigma_{\mathrm{T}}}{6 \pi m_{\mathrm{e}} c} B_{0}^{\prime 2}\left(c \Gamma / r_{0}\right)^{-2 b}
$$


Table 1 | Spectral parameters of models [e], [b], [f] and [g] (constant injection rate).

\begin{tabular}{lcllll} 
Model & $\boldsymbol{B}_{\mathbf{0}}^{\prime}(\mathbf{G})$ & $\boldsymbol{t}_{\text {obs }}(\mathbf{s})$ & $\boldsymbol{\alpha}$ & $\boldsymbol{\beta}$ & $\boldsymbol{E}_{\mathbf{p}}(\mathbf{k e V})$ \\
\hline$[\mathrm{e}]$ & 10 & 1 & -1.03 & -2.13 & 480 \\
{$[\mathrm{e}]$} & 10 & 3 & -1.03 & -2.10 & 220 \\
{$[\mathrm{~b}]$} & 30 & 1 & -1.22 & -2.26 & 490 \\
{$[\mathrm{~b}]$} & 30 & 3 & -1.17 & -2.26 & 220 \\
{$[\mathrm{f}]$} & 100 & 1 & -1.42 & -2.33 & 590 \\
{$[\mathrm{f}]$} & 100 & 3 & -1.39 & -2.35 & 240 \\
{$[\mathrm{~g}]$} & 300 & 1 & -1.50 & -2.34 & 650 \\
{$[\mathrm{~g}]$} & 300 & 3 & -1.50 & -2.37 & 250
\end{tabular}

We then find the solution of electron Lorentz factor at any time $t_{j}^{\prime}\left(>t_{i}^{\prime}\right)$

$$
\gamma_{\mathrm{e}}\left(t_{j}^{\prime}\right)=\left[\frac{1}{\gamma_{\mathrm{e}}\left(t_{i}^{\prime}\right)}+\frac{a}{1-2 b}\left\{t_{j}^{\prime 1-2 b}-t_{i}^{\prime 1-2 b}\right\}\right]^{-1}
$$

where $\gamma_{\mathrm{e}}\left(t_{i}^{\prime}\right)$ is the electron Lorentz factor at an initial time $t_{i}^{\prime}$. For $b>1 / 2, t_{j}^{\prime} \gg t_{i}^{\prime}$, and $\gamma_{\mathrm{e}}\left(t_{j}^{\prime}\right) \ll \gamma_{\mathrm{e}}\left(t_{i}^{\prime}\right)$, this solution gives $\gamma_{\mathrm{e}}\left(t_{j}^{\prime}\right) \propto t_{i}^{\prime 2 b-1}$. We then get

$$
\delta \gamma_{\mathrm{e}}\left(t_{j}^{\prime}\right) \propto t_{i}^{\prime 2 b-2} \delta t_{i}^{\prime} \propto\left[\gamma_{\mathrm{e}}\left(t_{j}^{\prime}\right)\right]^{\frac{2 b-2}{2 b-1}} \delta t_{i}^{\prime}
$$

For a constant injection rate $R_{\text {inj }}$, we have $\delta N_{\mathrm{e}} \propto \delta t_{i}^{\prime}$. Thus, we have an asymptotic behaviour of the global electron spectrum as follows.

$$
\frac{\delta N_{\mathrm{e}}}{\delta \gamma_{\mathrm{e}}\left(t_{j}^{\prime}\right)} \propto\left[\gamma_{\mathrm{e}}\left(t_{j}^{\prime}\right)\right]^{-\frac{2 b-2}{2 b-1}}
$$

Now we consider the full equation (2) that includes the adiabatic term. For $B^{\prime}(r)=B_{0}^{\prime}\left(r / r_{0}\right)^{-b}$ and $r=c t^{\prime} \Gamma$, equation (2) can be written as

$$
\frac{\mathrm{d}}{\mathrm{d} t^{\prime}}\left(\frac{1}{\gamma_{\mathrm{e}}}\right)=a t^{\prime-2 b}+\frac{2}{3 t^{\prime}}\left(\frac{1}{\gamma_{\mathrm{e}}}\right)
$$

This equation has an analytic solution

$$
\gamma_{\mathrm{e}}\left(t^{\prime}\right)=t^{\prime-2 / 3}\left[\frac{3 a}{1-6 b} t^{\prime(1-6 b) / 3}+C\right]^{-1}
$$

where $C$ is the integration constant of the differential equation, to be determined by the initial condition; $\gamma_{\mathrm{e}}\left(t_{i}^{\prime}\right)$ at time $t_{i}^{\prime}$. The electron's Lorentz factor $\gamma_{\mathrm{e}}\left(t_{j}^{\prime}\right)$ at a later time $t_{j}^{\prime}$ is found to be

$$
\gamma_{\mathrm{e}}\left(t_{j}^{\prime}\right)=t_{j}^{\prime-2 / 3}\left[\frac{t_{i}^{\prime-2 / 3}}{\gamma_{\mathrm{e}}\left(t_{i}^{\prime}\right)}+\frac{3 a}{1-6 b}\left\{t_{j}^{\prime(1-6 b) / 3}-t_{i}^{\prime(1-6 b) / 3}\right\}\right]^{-1}
$$

For $b>1 / 6, t_{j}^{\prime} \gg t_{i}^{\prime}$ and $t_{j}^{\prime 2 / 3} \gamma_{\mathrm{e}}\left(t_{j}^{\prime}\right) \ll t_{i}^{\prime 2 / 3} \gamma_{\mathrm{e}}\left(t_{i}^{\prime}\right)$, this solution gives $\gamma_{\mathrm{e}}\left(t_{j}^{\prime}\right) \propto t_{j}^{\prime-2 / 3} t_{i}^{\prime(6 b-1) / 3}$. A variation in $\gamma_{\mathrm{e}}\left(t_{j}^{\prime}\right)$ results only from $\delta t_{i}^{\prime}$ for the instantaneous (that is, at a fixed time $t_{j}^{\prime}$ ) global electron spectrum; $\delta \gamma_{\mathrm{e}}\left(t_{j}^{\prime}\right) \propto t_{j}^{\prime-2 / 3} t_{i}^{\prime(6 b-4) / 3} \delta t_{i}^{\prime}$. This gives the asymptotic behaviour of the global electron spectrum

$$
\frac{\delta N_{\mathrm{e}}}{\delta \gamma_{\mathrm{e}}\left(t_{j}^{\prime}\right)} \propto t_{j}^{\prime \frac{2}{6 b-1}}\left[\gamma_{\mathrm{e}}\left(t_{j}^{\prime}\right)\right]^{-\frac{6 b-4}{6 b-1}}
$$

where we have again assumed a constant injection rate $R_{\text {inj }}$. Therefore, we have the asymptotic low-energy electron spectral index $\tilde{p}_{a}=(6 b-4) /(6 b-1)$.

Dependence on other parameters. The 'normalization' factor $B_{0}^{\prime}$ is essential in defining the strength of magnetic fields seen by an electron during the cooling process since injection. So far, we have adopted the value $B_{0}^{\prime}=30 \mathrm{G}$. In the following, we calculate the cases for $B_{0}^{\prime}=10,100,300 \mathrm{G}$ for $b=1$ and constant injection rate (models [e], [f] and [g], respectively). To compare with model [b] $\left(B_{0}^{\prime}=30 \mathrm{G}\right.$ and $\left.b=1\right)$, we keep $\Gamma=300$ fixed and the product of $\gamma_{m}^{2} B_{0}^{\prime}$ as a constant to ensure the same observed $E_{\mathrm{p}}$. We then repeat the calculations as described in the main text and perform the Band function fits to the model spectra. The resulting Band function parameters are presented in Table 1. One can see that an $\alpha$ value ranging from $\sim-1$ to -1.5 is obtained. The general trend is that a lower $B^{\prime}$ tends to give rise to a harder $\alpha$ value.

The light curves of GRBs show erratic variability, and can be decomposed as the superposition of many 'pulses'. For a clean pulse, the decay phase of a pulse would be controlled by the high-latitude 'curvature' effect ${ }^{32}$. GRB light curves are typically composed of superposed pulses, so that the decay phase can be contaminated by the rising phase of an adjacent pulse. A test of the curvature
Table 2 | Spectral parameters of models [e1], [b1], [f1] and [g1] (linear increase of injection rate).

\begin{tabular}{llllll} 
Model & $\boldsymbol{B}_{\mathbf{0}}^{\prime}(\mathbf{G})$ & $\boldsymbol{t}_{\text {obs }}(\mathbf{s})$ & $\boldsymbol{\alpha}$ & $\boldsymbol{\beta}$ & $\boldsymbol{E}_{\mathbf{p}}(\mathrm{keV})$ \\
\hline$[\mathrm{e} 1]$ & 10 & 1 & -0.92 & -2.09 & 460 \\
{$[\mathrm{e} 1]$} & 10 & 3 & -0.92 & -2.07 & 220 \\
{$[\mathrm{~b} 1]$} & 30 & 1 & -1.13 & -2.24 & 460 \\
{$[\mathrm{~b} 1]$} & 30 & 3 & -1.09 & -2.23 & 210 \\
{$[\mathrm{f} 1]$} & 100 & 1 & -1.37 & -2.32 & 520 \\
{$[\mathrm{f} 1]$} & 100 & 3 & -1.34 & -2.33 & 220 \\
{$[\mathrm{~g} 1]$} & 300 & 1 & -1.48 & -2.33 & 610 \\
{$[\mathrm{~g} 1]$} & 300 & 3 & -1.48 & -2.35 & 240
\end{tabular}

Table 3 | Spectral parameters of models [e], [e1], [e2] and [e3] $\left(B_{0}^{\prime}=10 \mathrm{G}\right)$.

\begin{tabular}{llllll} 
Model & $\boldsymbol{q}$ & $\boldsymbol{t}_{\text {obs }}(\mathbf{s})$ & $\boldsymbol{\alpha}$ & $\boldsymbol{\beta}$ & $\boldsymbol{E}_{\mathbf{p}}(\mathbf{k e V})$ \\
\hline$[\mathrm{e}]$ & 0 & 1 & -1.03 & -2.13 & 480 \\
{$[\mathrm{e}]$} & 0 & 3 & -1.03 & -2.10 & 220 \\
{$[\mathrm{e} 1]$} & 1 & 1 & -0.92 & -2.09 & 460 \\
{$[\mathrm{e} 1]$} & 1 & 3 & -0.92 & -2.07 & 220 \\
{$[\mathrm{e} 2]$} & 2 & 1 & -0.87 & -2.06 & 470 \\
{$[\mathrm{e} 2]$} & 2 & 3 & -0.87 & -2.03 & 220 \\
{$[\mathrm{e} 3]$} & 3 & 1 & -0.82 & -2.04 & 470 \\
{$[\mathrm{e} 3]$} & 3 & 3 & -0.82 & -2.01 & 220
\end{tabular}

effect model of GRB pulses suggested that only about $40 \%$ of GRB pulses satisfy the model constraints ${ }^{33}$. Nonetheless, because the curvature effect only introduces $E_{\mathrm{p}}$ evolution but does not apparently modify the $\alpha$ value, in general the observed $\alpha$ values would be mostly defined by the rising history of electron injection. An increase in the injection rate with time gives more weight to electrons that are injected later, which tend to harden the spectrum. We test how the injection history during the rising phase affects $\alpha$. First, we introduce a linear increase of the injection rate for $B_{0}^{\prime}=10,30,100,300 \mathrm{G}$, respectively (with $b=1$ ) and name the models as [e1], [b1], [f1] and [g1], respectively. The fitted spectral parameters of these models are presented in Table 2 . One can see that by introducing a rise of injection rate with time, the resulting $\alpha$ values are systematically harder. For the four models discussed, the $\alpha$ value ranges from -0.92 to -1.48 .

The rising phase may be steeper than a linear increase with time. We next test the effect of different rising profiles on $\alpha$. We fix $B_{0}^{\prime}=10 \mathrm{G}$ to check how hard a spectrum one may get. Considering the injection rate $R_{\text {inj }}\left(t^{\prime}\right) \propto t^{\prime q}$, we calculate the cases for $q=0,1,2,3$ (models [e], [e1], [e2] and [e3], respectively). Table 3 shows the spectral parameters of these models. One can see that $\alpha$ hardens as $q$ increases (a more rapid increase). For these four models, the $\alpha$ value is in the range between -0.82 and -1.03 .

Received 8 March 2013; accepted 26 February 2014; published online 6 April 2014

\section{References}

1. Sari, R., Piran, T. \& Narayan, R. Spectra and light curves of Gamma-ray burst afterglows. Astrophys. J. 497, L17-L20 (1998).

2. Band, D. et al. BATSE observations of gamma-ray burst spectra. I-Spectral diversity. Astrophys. J. 413, 281-292 (1993).

3. Preece, R. D. et al. The BATSE gamma-ray burst spectral catalog. I. High time resolution spectroscopy of bright bursts using high energy resolution data. Astrophys. J. Supp. 126, 19-36 (2000).

4. Zhang, B-B. et al. A comprehensive analysis of Fermi gamma-ray burst data. I. Spectral components and the possible physical origins of LAT/GBM GRBs. Astrophys. J. 730, 141 (2011).

5. Nava, L., Ghirlanda, G., Ghisellini, G. \& Celloti, A. Spectral properties of 438 GRBs detected by Fermi/GBM. Astron. Astrophys. 530, A21 (2011).

6. Mészáros, P., Rees, M. J. \& Papathanassiou, H. Spectral properties of blast-wave models of gamma-ray burst sources. Astrophys. J. 432, 181-193 (1994). 
7. Daigne, F. \& Mochkovitch, R. Gamma-ray bursts from internal shocks in a relativistic wind: Temporal and spectral properties. Mon. Not. R. Astron. Soc. 296, 275-286 (1998).

8. Ghisellini, G., Celotti, A. \& Lazzati, D. Constraints on the emission mechanisms of gamma-ray bursts. Mon. Not. R. Astron. Soc. 313, L1-L5 (2000).

9. Pe'er, A. \& Zhang, B. Synchrotron emission in small-scale magnetic fields as a possible explanation for prompt emission spectra of gamma-ray bursts. Astrophys. J. 653, 454-461 (2006).

10. Derishev, E. V. Synchrotron emission in the fast cooling regime: Which spectra can be explained? Astrophys. Spa. Sci. 309, 157-161 (2007).

11. Zhao, X. et al. Gamma-ray burst spectrum with decaying magnetic field. Astrophys. J. 780, 12 (2014).

12. Asano, K. \& Teresawa, T. Slow heating model of gamma-ray burst: Photon spectrum and delayed emission. Astrophys. J. 705, 1714-1720 (2009).

13. Burgess, J. M. et al. Constraints on the synchrotron shock model for the Fermi GRB 090820A observed by gamma-ray burst monitor. Astrophys. J. 741, 24 (2011).

14. Burgess, J. M. et al. Time-resolved analysis of Fermi GRBs with fast and slow-cooled synchrotron photon models. Astrophys. J. 784, 17 (2014).

15. Rybicki, G. B. \& Lightman, A. P. Radiative Processes in Astrophysics (Wiley-Interscience, 1979).

16. Spruit, H. C., Daigne, F. \& Drenkhahn, G. Large scale magnetic fields and their dissipation in GRB fireballs. Astron. Astrophys. 369, 694-705 (2001)

17. Rees, M. J. \& Mészáros, P. Unsteady outflow models for cosmological gamma-ray bursts. Astrophys. J. 430, L93-L96 (1994)

18. Daigne, F., Bošnjak, Z. \& Dubus, G. Reconciling observed gamma-ray burs prompt spectra with synchrotron radiation? Astron. Astrophys. 526, A110 (2011)

19. Zhang, B. \& Yan, H. The internal-collision-induced magnetic reconnection and turbulence (ICMART) model of gamma-ray bursts. Astrophys. J. 726, 90 (2011).

20. Uhm, Z. L. et al. Dynamics and afterglow light curves of gamma-ray burst blast waves with a long-lived reverse shock. Astrophys. J. 761, 147 (2012).

21. Gao, H., Zhang, B-B. \& Zhang, B. Stepwise filter correlation method and evidence of superposed variability components in gamma-ray burst prompt emission light curves. Astrophys. J. 748, 134 (2012).

22. Lu, R-J. et al. A comprehensive analysis of Fermi gamma-ray burst data. II. $E_{\mathrm{p}}$ evolution patterns and implications for the observed spectrum-luminosity relations. Astrophys. J. 756, 112 (2012).

23. Guiriec, S. et al. Detection of a thermal spectral component in the prompt emission of GRB 100724B. Astrophys. J. 727, L33 (2011).

24. Axelsson, M. et al. GRB110721A: An extreme peak energy and signatures of the photosphere. Astrophys. J. 757, L31 (2012).
25. Guiriec, S. et al. Evidence for a photospheric component in the prompt emission of the short GRB 120323A and its effects on the GRB hardness-luminosity relation. Astrophys. J. 770, 32 (2013).

26. Zhang, B., Lu, R-J., Liang, E-W. \& Wu, X-F. GRB 110721A: Photosphere 'Death Line' and the physical origin of the GRB Band function. Astrophys. J. 758, L34 (2012).

27. Veres, P., Zhang, B-B. \& Mészáros, P. The extremely high peak energy of GRB $110721 \mathrm{~A}$ in the context of a dissipative photosphere synchrotron emission model. Astrophys. J. 761, L18 (2012).

28. Mészáros, P. \& Rees, M. J. Steep slopes and preferred breaks in gamma-ray burst spectra: The role of photospheres and comptonization. Astrophys. J. 530, 292-298 (2000).

29. Pe’er, A., Mészáros, P. \& Rees, M. J. The observable effects of a photospheric component on GRB and XRF prompt emission spectrum. Astrophys. J. 642 995-1003 (2006).

30. Beloborodov, A. M. Collisional mechanism for gamma-ray burst emission. Mon. Not. R. Astron. Soc. 407, 1033-1047 (2010).

31. Lazzati, D., Morsony, B. J., Margutti, R. \& Begelman, M. C. Photospheric emission as the dominant radiation mechanism in long-duration gamma-ray bursts. Astrophys. J. 765, 103 (2013).

32. Kumar, P. \& Panaitescu, A. Afterglow emission from naked gamma-ray bursts. Astrophys. J. 541, L51-L54 (2000).

33. Kocevski, D., Ryde, F. \& Liang, E. Search for relativistic curvature effects in gamma-ray burst pulses. Astrophys. J. 596, 389-400 (2003)

\section{Acknowledgements}

This work is partially supported by the China Postdoctoral Science Foundation through Grant No. 2013M540813, the National Basic Research Program ('973' Program) of China under Grant No. 2014CB845800, and by the National Science Foundation under grant AST-0908362. We acknowledge helpful discussion with the following colleagues:

A. Beloborodov, F. Daigne, G. Ghisellini, K. Ioka, P. Kumar, D. Lazzati, Z. Li, P. Mészáros, K. Murase, R. Mochkovitch and A. Pe'er.

\section{Author contributions}

Z.L.U. wrote the numerical code, performed the analytical and numerical calculations, and prepared the figures. Both authors conceived the project. B.Z. wrote the manuscript

\section{Additional information}

Reprints and permissions information is available online at www.nature.com/reprints Correspondence and requests for materials should be addressed to B.Z.

\section{Competing financial interests}

The authors declare no competing financial interests. 